\title{
A novel biomarker for early stage ovarian cancer, and a new target for immunotherapy?
}

\author{
Ghazala Khan ${ }^{1}$, Kelly Grayson ${ }^{1}$, Suzanne Brooks ${ }^{2}$, Ken Mills ${ }^{3}$ and Barbara Guinn ${ }^{*}$ \\ ${ }^{1}$ Department of Biomedical Sciences, University of Hull, UK. \\ 2Biomedical Imaging Unit, Southampton University Hospitals Trust, Southampton, UK. \\ ${ }^{3}$ Centre for Cancer Research and Cell Biology, Queens' University Belfast, UK \\ *b.guinn@hull.ac.uk
}

\begin{abstract}
Introduction
Ovarian cancer (OC) affects approximately 7,500 women in the U.K. every year. Although it can occur at any age it is most common after menopause. Early diagnosis significantly improves the chances of effective treatment however diagnosis tends to be in the later stages of disease when treatment options are more limited. Currently diagnosis is confirmed by a pelvic examination, transvaginal ultrasonography and detection of carbohydrate antigen 125 (CA125) in the tumour tissue. However CA125 has variable expression between patients with some expression in endometrial tissue. Human epididymis secretory protein 4 (HE4) is overexpressed in ovarian cancers but lacks specificity with similar sensitivity to CA125. Both work best as part of a panel of biomarkers for the detection of OC. There still remains a need to identify a singularly good biomarker which could positively influence disease outcome by enabling early detection. To this end we investigated the expression of OCP, a cancer-testis antigen known to have expression restricted to cancer and MHC class I negative cells.
\end{abstract}

\section{Materials and Methods}

Immunohistochemistry. Paraffin-embedded ovarian cancer tissue microarrays containing 195 ovarian cancer samples, 12 healthy ovarian tissues and 1 skin cancer sample (Biomax) were de-waxed and prepared for antigen retrieval using 50mM Tris-EDTA at pH9.0. We used actin as a positive control, and cells only and isotype controls to detect nonspecific staining. We compared staining to that achieved with CA125, the current standard marker for ovarian cancer, WT1 and HE4. Immunolabelling for each of the antigens was performed using antihuman primary antibodies and HRP conjugated secondary antibodies (all Abcam, U.K.) Sub-cellular localisation of proteins under test were visualised through the use of the Envision-HRP labelling kit (Dako, U.K.).

\section{Results}

We examined ovarian cancer protein (OCP) expression in a number of cell lines and found that Skov3, OVCAR3, A2780 and HeLa all expressed OCP. We showed that the expression of the anti-OCP antibody could be blocked by OCP-specific peptides (Figure 1).

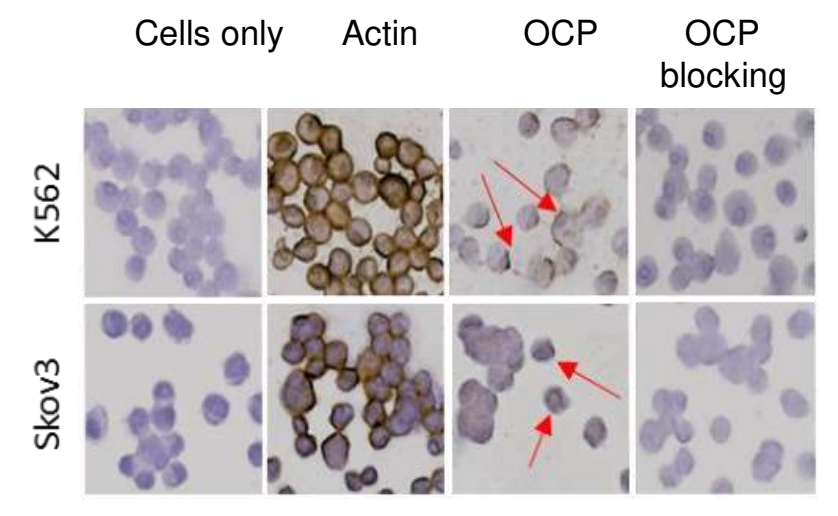

Figure 1. OCP antigen immunolabelling was visible as a brown deposit. Actin was used as a positive control and cells only and isotype matched antibodies were used as controls for non-specific staining. Peptide blocking prevented immunolabelling of OCP suggesting that labelling is specific.

We examined patient samples and found a higher staining intensity and a more frequent expression of OCP in early stage OC than found when using CA125, HE4 or WT1 (Figures 2 and 3). In addition the expression of OCP was specific to OC and was not found in normal ovarian, normal adjacent, endometrial or inflamed tissue.

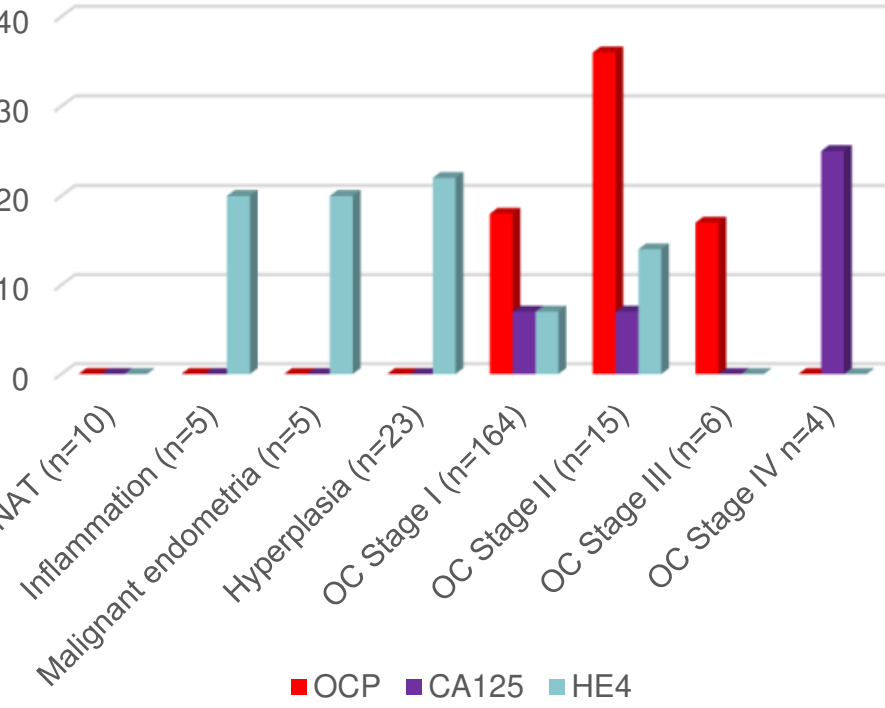

Figure 2. OCP antigen expression was directly compared to other well known antigens in OC. Although OCP was not detected in healthy or disease related tissues, it was found in stages I, II and III of OC. This frequency of detection and specificity was not seen with the other antigens tested (CA125, HE4 or WT1).

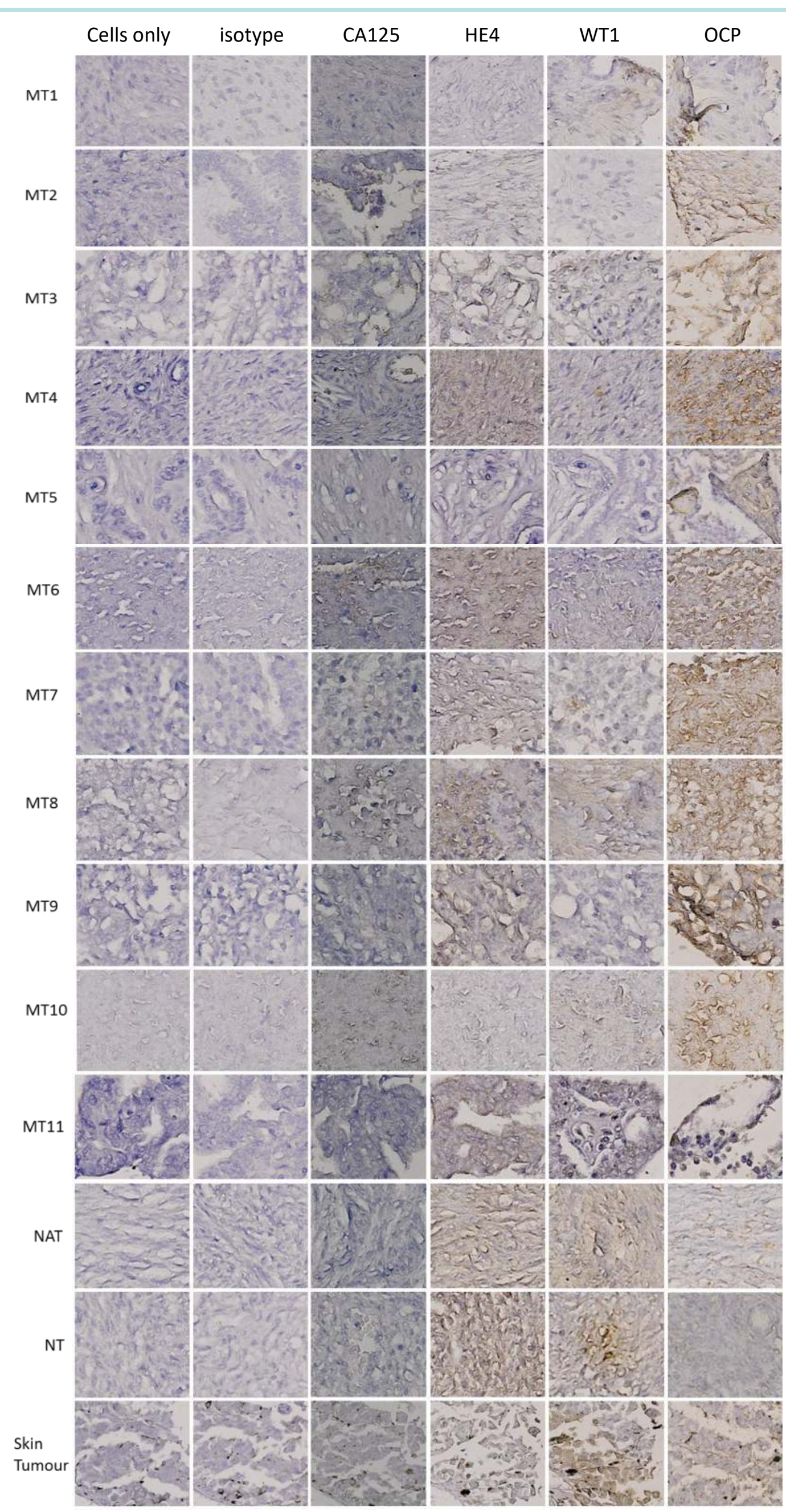

Figure 3. Immunolabelling of OCP was at a higher intensity and more frequent in OC patients when compared with CA125, HE4 or WT1. Most samples analysed were from stage I (MT1-10, in this image) and II (MT11, in this image) disease. We also found no immunolabelling of OCP in normal donors and normal adjacent tissue. Cells only and isotype antibodies were used as negative controls for immunolabelling.

\section{Summary}

- We have identified OCP as a possible biomarker of early stage OC.

- OCP has a number of family members but we have identified an isoform that is specifically overexpressed in OC

- OCP shows increased sensitivity and specificity for stage I and II OC when compared to the current biomarkers - CA125 and HE4

- $\mathrm{OCP}$ is a cancer-testes antigen that is already known to have restricted expression in healthy tissues

\section{Future Directions}

- OCP expression will be examined in fresh-frozen tissues to determined whether we can reduce non-specific binding and improve sensitivity of detection

- $\quad$ Antibodies will be validated to demonstrate their specificity using cell lines transfected with OCP 